\title{
Main Predictors of Necrotizing Periodontal Diseases: A Review
}

Pâmela Gama de Freitas ${ }^{1}$, Helaine Cristina da Costa Vieira ${ }^{1}$, Natália de Oliveira ${ }^{1}$, Camila Abreu Carvalho dos Santos ${ }^{1}$, Idiberto José Zotarelli Filho ${ }^{2}$, Leandro Moreira Tempest ${ }^{1,2}$ and Patrícia Garani Fernandes ${ }^{1,2}$

1 University Center North Paulista (Unorp) - São José do Rio Preto - SP, Brazil

${ }^{2}$ Post Graduate And Continuing Education (Unipos), Street Ipiranga, 3460, São José do Rio Preto SP, Brazil

*Corresponding author: Idiberto José Zotarelli Filho, Unipos - Post Graduate And Continuing Education, Street Ipiranga, 3460, São José do Rio Preto SP, Brazil, Tel: +55(17) 98166-6537 / +55(17) 98803-7459; E-mail: m.zotarelli@gmail.com

Received date: March 14, 2018; Accepted date: March 22, 2018; Published date: March 29, 2018

Copyright: ( 2018 Freitas PG, et al. This is an open-access article distributed under the terms of the Creative Commons Attribution License, which permits unrestricted use, distribution, and reproduction in any medium, provided the original author and source are credited.

\section{Abstract}

Background: Necrotizing periodontal diseases rapidly and aggressively reach the periodontal tissues and are multifactorial and complex. Necrotizing ulcerative gonorrhea (GUN) necrotizing periodontitis (PUN) and necrotizing ulcerative stomatitis (EUN) are the three main modalities of necrotizing periodontal diseases.

Objective: was to report through a literature review the necrotizing periodontal diseases, highlighting its manifestation, diagnosis and means of more conservative treatments, thus reducing the sequelae caused by the disease.

Methods: Experimental and clinical studies were included (case reports, retrospective, prospective and randomized trials) with qualitative and / or quantitative analysis. A total of 30 articles were found involving Periodontal Diseases, Necrotizing Periodontal Diseases, Oral Health, Diabetes mellitus. A total of 21 articles were evaluated in full, and 13 were included and discussed in this study.

Conclusion: It is concluded that necrotizing periodontal diseases are diseases of few occurrences, but are considered the most aggressive form of periodontal diseases. Biofilm is the main cause, but it has to take into account the predisposing factors to have a better diagnosis.

Keywords Periodontal diseases; Necrotizing periodontal diseases; Oral health; Diabetes Mellitus

\section{Introduction}

Necrotizing periodontal diseases rapidly and aggressively reach the periodontal tissues and are multifactorial and complex [1]. Necrotizing ulcerative gonorrhea (GUN) necrotizing periodontitis (PUN) and necrotizing ulcerative stomatitis (EUN) are the three main modalities of necrotizing periodontal diseases [2]. Microscopically, necrotizing periodontal diseases are characterized by a superficial infiltration of polymorphonuclear neutrophils (PMN) consisting mainly of lymphocytes and plasma cells $[1,2]$.

Clinically they present with ulceration, necrosis, pain, spontaneous or touch bleeding, odor, metallic taste and the patient may have a fever [3]. The accumulation of biofilm is the main cause of the disease, but some predisposing factors can trigger the disease, for example: malnutrition, high stress, smoking, local trauma, patients with immunodeficiency among others [4].

GUN is an acute bacterial infection that affects the protective tissues of the tooth. Clinically it presents a limited gingival necrosis, gingival bleeding, neurotic ulcers, odor and excess saliva. The treatment should be performed with light movements removing the biofilm, instructing the patient to improve hygiene, the use of a local antibiotic such as $0.12 \%$ chlorhexidine and monitoring of the disease until its regression may be prescribed [5].
PUN exhibits areas of tissue necrosis with loss in the supporting tissues and has formation of periodontal pockets. The treatment aims to control and regress the symptoms of the disease, to remove biofilm, scaling and root planing, to perform local debridement and the removal of necrotic remains, and the use of local and systemic antibiotics, chlorhexidine $0.12 \%$ and metronidazole [6].

EUN is considered to be the most severe of the periodontal diseases it destroys soft and hard tissues in the orofacial region [7]. It affects mainly children from 2 to 16 years old and has higher prevalence in poor countries and with malnutrition. It is common for the patient to have fever and anemia, so rehydration should be performed in addition to prescribing a high-protein diet. Treatment with systemic and local antibiotics is of extreme necessity, such as chlorhexidine $0.12 \%$ and metronidazole. Necrotic tissues should be removed by scaling root planing and irrigation with povedine at $10.0 \%$ [8].

The objective of this study was to report through a literature review the necrotizing periodontal diseases, highlighting its manifestation, diagnosis and means of more conservative treatments, thus reducing the sequelae caused by the disease.

\section{Methods}

Experimental and clinical studies were included (case reports, retrospective, prospective and randomized trials) with qualitative and / or quantitative analysis. Initially, the key words were determined by searching the DeCS tool (Descriptors in Pubmed, Health Sciences, BIREME base) and later verified and validated by MeSh system 
Page 2 of 3

(Medical Subject Headings, the US National Library of Medicine) in order to achieve consistent search.

\section{Mesh Terms}

The words were included Periodontal Diseases, Necrotizing Periodontal Diseases, Oral Health, Diabetes mellitus. The literature search was conducted through online databases: Pubmed, Periodicos.com and Google Scholar. It was stipulated deadline, and the related search covering all available literature on virtual libraries.

\section{Series of Articles And Eligibility}

A total of 30 articles were found involving Periodontal Diseases, Necrotizing Periodontal Diseases, Oral Health, Diabetes mellitus. Initially, it was held the exclusion existing title and duplications in accordance with the interest described this work. After this process, the summaries were evaluated and a new exclusion was held. A total of 21 articles were evaluated in full, and 13 were included and discussed in this study.

\section{Literature Review}

\section{Necrotizing ulcerative gingivitis (GUN)}

Necrotizing ulcerative gingivitis is unique among periodontal diseases. An altered ability to deal with psychological stress, immunosuppression, and smoking has been strongly associated with the onset of the disease. It is a painful bacterial infection that affects the interdental and marginal gingival tissues. It is distinct from other periodontal diseases, in that it presents clinically due to interdental gingival necrosis, gingival hemorrhage and pain [9]. A patient with GUN almost always has complaints of pain or discomfort, which may have had a rapid onset [10].

The manipulation of the affected areas can be very painful and the patient may show some resistance to the periodontal probing of the affected sites $[10,11]$. Invariably, there is ulceration of the interdental papillae. Ulcerations, which may extend to the marginal gingiva, may result in the reduction of the adhered gingival band due to marginal necrosis [12].

Characteristically, ulcers are crateriform and the term "inverted" has been used to describe the interdental papillae. If intact, the ulcerated surface is usually covered by a layer of necrotic debris, also referred to as "pseudomembrane" [13]. This is composed of fibrin, necrotic tissue, inflammatory cells and masses of dead or still viable bacteria, and is rapidly detached, exposing hemorrhagic ulceration in the underlying tissues. The area between the margin of necrosis and the unaffected gingival tissue usually presents with a narrow erythematous band, also classified as linear erythema [12].

\section{Necrotizing ulcerative periodontitis (PUN)}

According to the 1992 Glossary of Periodontal Terms, necrotizing ulcerative periodontitis is defined as "a disease of rapid and severe progression that has a distinct erythema in the free gingiva, adherent gingiva, and alveolar mucosa; There is extensive necrosis of soft tissue and loss of severe periodontal insertion. The formation of deep pockets is not evident " It may also be defined as "an infection characterized by necrosis of the gingival tissues, periodontal ligament, and alveolar bone." [10]. These lesions are most frequently observed in systemically compromised individuals, including HIV infection, severe malnutrition, and immunosuppression.

There is no evidence to support the concept that PUN is the natural progression of GUN, other than the occurrence of accidental insertion loss resulting from interdental soft tissue destruction associated with GUN [13]. Detection of insertion loss associated with or as a consequence of GUN does not confer the need for a diagnosis of PUN, especially in patients who are known to be systemically healthy and non-immunocompromised seronegatives for HIV [13].

\section{Necrotizing ulcerative stomatitis}

Necrotizing ulcerative stomatitis is considered to be a late stage of necrotizing periodontal disease. It results from the extent of necrosis to the alveolar mucosa, that is, when necrosis Necrotizing Periodontal Disease exceeds the mucogingival line [11-13]. Necrotizing ulcerative stomatitis can result in extensive bone exposure, with the formation of large sequestrations, and consequently the development of oro-antral fistulas and osteitis, also called cancrum oris, is a serious, opportunistic and devastating infectious disease characterized by tissue destruction soft and hard areas of the orofacial region [11].

Although occurring all over the world, noma cases are currently less reported in developed countries [12]. However, it remains a prevalent disease, particularly in Sub-Saharan Africa, where poverty, malnutrition and preventable childhood infections are still widespread. The annual global incidence is about 140,000 cases and the mortality rate exceeds $90.0 \%$ in the absence of timely treatment. It mainly affects children between 2 and 16 years of age, with a peak incidence between 3 and 6 years. Both sexes are equally affected. In later stages it can occur in adolescents and adults [12].

\section{Treatments}

Current treatment As a consequence of the acute clinical manifestations of these diseases, it is necessary to introduce some modifications to the general conventional treatment of periodontal disease induced by bacterial plaque and to complement it with certain therapeutic measures, which aim at the early treatment of the disease [13]. The treatment of necrotizing periodontal disease can be schematized in four phases: emergency treatment; Treatment or elimination of predisposing factors; Surgical correction of tissue deformities; maintenance [13].

\section{Discussion}

Necrotizing periodontal disease is the generic term that encompasses a set of serious, rapidly destructive, or even potentially fatal pathologies [12]. Although rare in developed countries, noma is the most severe and advanced stage of the disease. In its pathogenesis, it can develop from a pre-existing necrotizing ulcerative gingivitis and after infection by Fusobacterium necrophorum and Prevotella intermedia.

The diagnosis of these diseases can be made only on the basis of their clinical characteristics and is fundamental, not only because they contribute to the loss of periodontal insertion and gingival sequelae, but also because they are a marker of immune deterioration in patients who are HIV positive [12]. Although its etiology is not fully understood, spindle bacteria and spirochetes and a decrease in host systemic resistance are required to initiate the disease process. During the acute phase of the disease, the administration of antibiotics, such as 
metronidazole, is often necessary for ulcer healing and pain relief to allow the initiation of non-surgical periodontal treatment [13].

The control or elimination of predisposing factors, the surgical correction of tissue defects and periodic maintenance appointments are extremely important to avoid relapse of the disease and the consequent progression of the destruction of the supporting periodontal tissues $[12,13]$.

\section{Conclusion}

It is concluded that necrotizing periodontal diseases are diseases of few occurrences, but are considered the most aggressive form of periodontal diseases. Biofilm is the main cause, but it has to take into account the predisposing factors to have a better diagnosis. And the faster the disease is detected, the better the prognosis and thereby lesser occurrence of the sequelae caused by the disease. We should advise the patient that the best treatment is to maintain good oral hygiene, with good brushing and flossing regularly.

\section{Competing Interests}

The authors none declare.

\section{References}

1. Albandar JM, Tinoco EB (2002) Global epidemiology of periodontal diseases in children and young persons. Periodontol 2000 29: 153-176.

2. Bascones-Martínez A, Escribano-Bermejo M (2005) Enfermedad periodontal necrosante: una manifestación de trastornos sistémicos. Med Clin 125: 706-713.
3. Chung CP, Nisengard RJ, Slots J, Genco RJ (1983) Bacterial IgG and IgM antibody titers in acute necrotizing ulcerative gingivitis. J Periodontol 54: 557-562.

4. Clerehugh V, Tugnait A (2001) Diagnosis and management of periodontal diseases in children and adolescents. Periodontol 2000 26: 146-168.

5. Cobb CM, Ferguson BL, Keselyak NT, Holt LA, MacNeill SR, et al. (2003) A TEM/SEM study of the microbial plaque overlying the necrotic gingival papillae of HIV-seropositive, necrotizing ulcerative periodontitis. J Periodontal Res 38: 147-155.

6. Enwonwu CO, Falkler WA Jr, Idigbe EO, Afolabi BM, Ibrahim M, et al. (1999) Pathogenesis of cancrum oris (noma): confounding interactions of malnutrition with infection. Am J Trop Med Hyg 60: 223-232.

7. Fabbri C, Fuller R, Bonfa E, Guedes LK, D’Alleva PS, et al. (2014) Periodontitis treatment improves systemic lupus erythematosus response to immunosuppressive therapy. Clin Rheumatol 33: 505-509.

8. Armitage GC (2004) Periodontal diagnoses and classification of periodontal diseases. Periodontol 2000 34: 9-21.

9. Page RC, Kornman KS (1997) The pathogenesis of human periodontitis: an introduction. Periodontol 2000 14: 9-11.

10. Silva TA, Garlet GP, Fukada SY, Silva JS, Cunha FQ (2007) Chemokines in oral inflammatory diseases: apical periodontitis and periodontal disease. J Periodontal Res 86: 306-319.

11. Brito RBD, Scarel-Caminaga RM, Trevilatto PC, Souza ABD, Barros SP (2004) Polymorphisms in the vitamin D receptor gene are associated with periodontal disease. J Periodontol 75: 1090-1095.

12. Offenbacher S (1996) Periodontal diseases: pathogenesis. Ann Periodontol 1: 821-878.

13. Kornman KS, Crane A, Wang HY, Giovine FSD, Newman MG, et al. (1997) The interleukin1 genotype as a severity factor in adult periodontal disease. J Clin Periodontol 24: 72-77. 